\title{
Behavioral Responses of Tabanidae and Stomoxys calcitrans to Unbaited and Baited Nzi and Horse Pal Traps in Southern Sweden
}

\author{
Gabriela Vaduva \\ Department of Biology, Lund University, Lund 22362, Sweden. \\ E-mail: vaduva_gabriela@yahoo.se
}

Received: August 31, 2020 Accepted: September 26, 2020

doi:10.5296/jbls.v11i2.17806 URL: https://doi.org/10.5296/jbls.v11i2.17806

\begin{abstract}
The distribution and species diversity of tabanids and stable flies, Stomoxys calcitrans Linnaeus, 1758, in the research area, Hästhult (wood pasture) in Sweden, as well as the behavioral responses of biting flies to visual and olfactory cues were highlighted by this study. Beyond the control of tabanids and stable flies through the Nzi and Horse Pal traps that act as a surrogate host, this study sought to demonstrate the attractiveness of biting flies to these traps by comparing the results of the two periods in which the traps were unbaited and baited. The odor attractants, such as urine (a mixture of more than phenol) and acetone used to increase trap yields, made the difference to the Horse Pal and Nzi traps for S. calcitrans (L) because the number of flies caught was significantly higher. Through this study, Nzi trap with bait and Horse Pal trap with and without bait were tested for the first time as regards the tabanids and S. calcitrans (L) in Scandinavia. Surprisingly, the number of tabanids caught in the Nzi trap was equal in both situations with or without bait. The result underlined the idea that the Nzi trap manages to catch a large number of tabanids even in the absence of attractants. It turns out that the Horse Pal trap was just as effective with and without bait in capturing tabanids. Furthermore, the number of tabanids caught in both traps depended on the type of weather, and the number of stable flies depended on the weather and also the type of trap. The results of this study showed that both the visual and olfactory aspects of the Nzi and Horse Pal traps are always essential, being used successfully in the control of biting flies and optimized for various research purposes.
\end{abstract}

Keywords: Attractants, Attractiveness, Horse Pal trap, Nzi trap, Species recognition, Stomoxys calcitrans, Tabanidae, Weather 


\section{Introduction}

Tabanids, commonly called horse flies and deer flies, and Stomoxys calcitrans Linnaeus, 1758 (Diptera) cause a great concern to the owners of beef cattle and dairy cows. These hematophagous species make the animals to suffer from loss of blood, loss of time for grazing and energy dissipated trying to evade their enemies. As a result, cattle milk production could easily be curtailed (Ndegwa and Ogodo, 2002). Moreover, the annoyance of tabanids interferes easily with livestock culture and human activities.

In tabanids, only the females suck blood and both sexes feed on a wide variety of nectar, honeydew and similar substances. Nectar is considered to be an important source of energy for tabanids, but their mechanism in nectar source locating is less known. Some species of tabanids are entirely feeders on nectar. In contrast, both sexes of S. calcitrans (L) are blood-feeding synanthropic pests. When the tabanids manage to get a full meal of blood, they go to a resting place for some days, waiting the blood to be digested (Wood, 1985). S. calcitrans (L) requires blood meal once every twenty-four hours and all the nutrients existing in nectar and pollen are going to keep the flies alive increasing their chances in getting the necessary blood meal (Allan et al., 1987; Hogsette et al., 1987). Without the blood meal, the eggs of some female biting flies cannot be matured. Likewise, tabanids and stable flies, $S$. calcitrans (L) freely movement from one animal to another trying to obtain a blood meal, resulting in a mechanical transmission of disease such as tularemia, anthrax and besnoitiosis a cattle disease (the causative agent, Besnoitia besnoiti is a cyst-forming coccidian parasite) widespread throughout Europe, Africa and Asia (Allan et al., 1987; Liénard et al., 2013; Vaduva, 2016). S. calcitrans (L) is a diurnal species, being less tied to livestock might travel widely in its searching of breeding sites and hosts (Wood, 1985).

It is an important vector of Trypanosoma evansi and Habronema microstoma, a horses' nematode (Wood, 1985; Mullens, 2002; Vaduva, 2016). A Stomoxys flies' outbreak in April and May 1997, led to the death of a large number of ungulates including Bongo over a large area of the tri - national region of Cameroon - Congo - Central African Republic (Kingdon, 1982). Furthermore, tabanids species, Haematopota pluvialis and Tabanus bromius are vectors of Trypanosoma theileri an important infection in cattle.

Tabanids are considered to be the mechanical vectors of thirsty five pathogenic agents including Anaplasma marginale, Trypanosoma vivax, Loa loa etc. (Foil and Hogsette, 1994; Vaduva, 2016). The most important diseases transmitted by deer flies to humans through repeated baits, are loiasis and tularemia (Mullens, 2002). Tabanids are nervous feeders tending to visit hosts repeatedly which give them a dangerous potential to move disease organisms quickly from host to host. The pathogen does not develop in the fly (Axtell, 1976). As well as, when it comes to transmitting a disease, relatively few human health problems are associated with deer flies and horse flies.

In attempts to monitor the species and number of biting flies, a wide variety of traps with different designs have been tested, obtaining more or less satisfactory results. However, Nzi and Horse Pal traps have proven to be practical and effective in capturing a large number of tabanids, stable flies and tsetse. Studies with Nzi trap have been made in North America, 
Australia, Africa and also in northern Skåne, Sweden (Vaduva, 2016). Horse Pal trap is considering to be an efficient trap by catching a plenty of horse flies in North America, where tabanids are particularly abundant, especially in forested regions. A comparative study between Epps and Horse Pal traps in central North Carolina pointed out that the second one captured a great number of tabanids and could be moved easily by one person (Watson et al., 2007).

There has been no previous research on the monitoring and establishment of appropriate monitoring techniques for tabanids and S. calcitrans (L) using baited Nzi trap and Horse Pal trap with and without bait in the vicinity of cows in Sweden. My published research work has been focused on the attractiveness of tabanids and stable flies to the unbaited Nzi trap tested in two different locations and in relation to cows and horses. The two previous locations, Gundrastorp and Kämlehöjalt, were in a straight line at a distance of $9.7 \mathrm{~km} \mathrm{NE}$ and respectively, $4.7 \mathrm{~km} \mathrm{NW}$ from the research area of this study. All information obtained through various studies is necessary, because there is no way to establish a local fauna list without knowledge in terms of daily pattern activity of different species of bloodsucking flies. Since tabanids and S. calcitrans (L) are considered to be vectors of different diseases, their populations must be controlled in attempt to reduce the pathogen transmission risk. Plenty of tabanids are diurnal feeders that look for their hosts primarily by sight which make them not to be effectively discouraged by insect repellents.

The study revealed the existence of 15 species of tabanids that together with $S$. calcitrans (L) can certainly be considered true pests in that area in Sweden.

The main objectives underlying this study were the following: a) the difference in the attractiveness of the Nzi trap with and without bait to both species of biting flies, tabanids and S. calcitrans (L); b) the same objective for the Horse Pal trap with and without bait and in addition, the possibility for this type of trap to attract $S$. calcitrans (L) to a greater or lesser extent; c) again the possibility that this type of trap, Horse Pal, might be introduced in the capture of biting flies, especially tabanids in this geographical area; d) are both types of traps, Nzi and Horse Pal equally effective in the catching of biting flies? Furthermore, the activity pattern of biting flies on all types of weather in relation to the type of trap with and without bait, including daily catches and temperature has been highlighted by this research.

\section{Research Methods}

\subsection{Traps}

The Nzi trap has been bought from Rincon - Vitova Insectaries company (USA) and used in daily monitoring of tabanids and S. calcitrans (L) (Figure 1).

It is made from flexible cloth marine grade canvas with an interesting combination of black, blue and white colors. Nzi trap works well in terms of biting flies' attractiveness to large blue and black objects. The dimensions of trap are as follows: 4.5 feet tall, 6 feet wide and 2 feet deep. The trap shape imitates large animals and the visual system of biting flies perceives the front blue panel as being the animal's "underbelly". The number of biting flies caught in this type of trap is high either with attractants or not. All the flies enter a collecting bottle 
positioned above directly through a funnel and then fall into a collecting bag where they eventually die because they cannot return. The effectiveness of this trap has been demonstrated in several places around the world, especially by catching at the same time several different types of biting flies such as tabanids, stable flies and tsetse (Mihok, 2002; Van Hennekeler et al., 2008; Vaduva, 2016).

In addition to the Nzi trap, one Horse Pal trap that was purchased from Newman Enterprises, Omro, WI 54963, USA was used in this study. This type of trap is made from premium materials such as marine grade canvas, epoxy coated steel legs, fiberglass screen, a shiny black ball and other materials for many seasons use. The biting flies are very good visual hunters and are attracted to this trap considering it a blood meal and end up being trapped in a clear big jar at the top of the escape route where they eventually decompose in sunlight. The size of trap is $0.61 \mathrm{~m}$ square and $1.68 \mathrm{~m}$ tall. Horse Pal trap requires no chemicals or messy baits and is designed specifically for horse flies, deer flies and yellow fly (Figure 2).

In the manufacturer's specifications, it was said, among other things, that this trap does not attract other types of flies, including stable flies. The effectiveness of Horse Pal trap in the catching of tabanids was demonstrating by a two summer seasons study made in North Carolina by Watson et al., (2007).

During the forty - two days, were performed two tests on both, Nzi and Horse Pal traps, in terms of the attractiveness for biting flies.

For the first twenty - one days, both types of traps were unbaited and over the next twenty one days, the efficiency of the attractant such as acetone (glass vial of $100 \mathrm{ml}$ ) and aged urine (glass vial of $500 \mathrm{ml}$ ) dug a little into the ground inside of each trap, sheltered from rain, in order to attract tabanids and S. calcitrans (L) has been tested.

Furthermore, two types of glass vials were used for each type of trap, containing each of them a different attractant such as acetone (as a long range orientation cue; purity 100\%) and urine that was aged for 15 days before use in order to develop the phenolic components. The odors were dispersed by a $6 \mathrm{~mm}$ aperture in diameter for acetone that lead to flow rate of $495 \mathrm{mg} / \mathrm{h}$ and a $45 \mathrm{~mm}$ diameter aperture for urine with a flow rate of $1000 \mathrm{mg} / \mathrm{h}$. When the situation required it, the 4 existing glass vials two by two inside the Nzi and Horse Pal traps and containing the two different attractants, acetone and aged urine, were refilled with the same type of bait. 


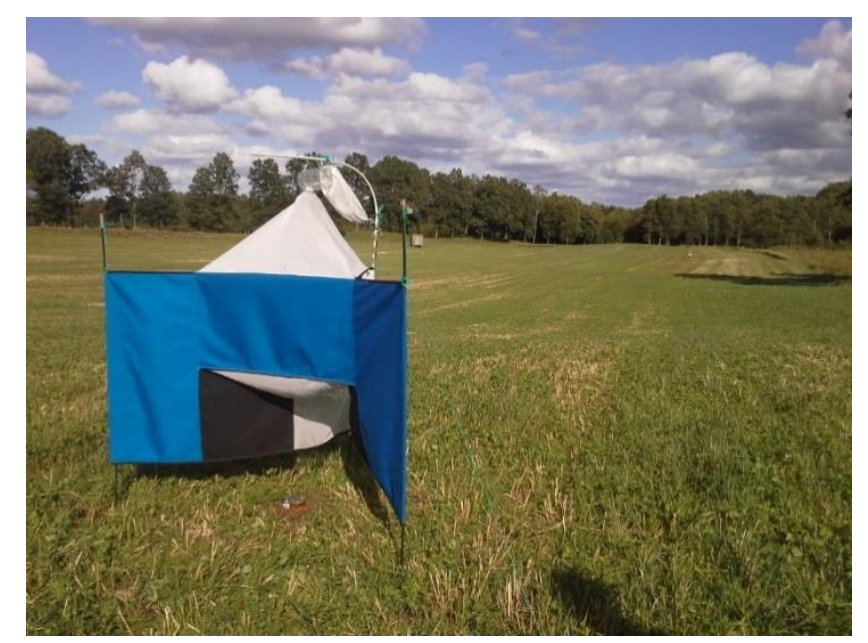

Figure 1. Baited Nzi trap set up in Hästhult (wood pasture), Sweden, 2016

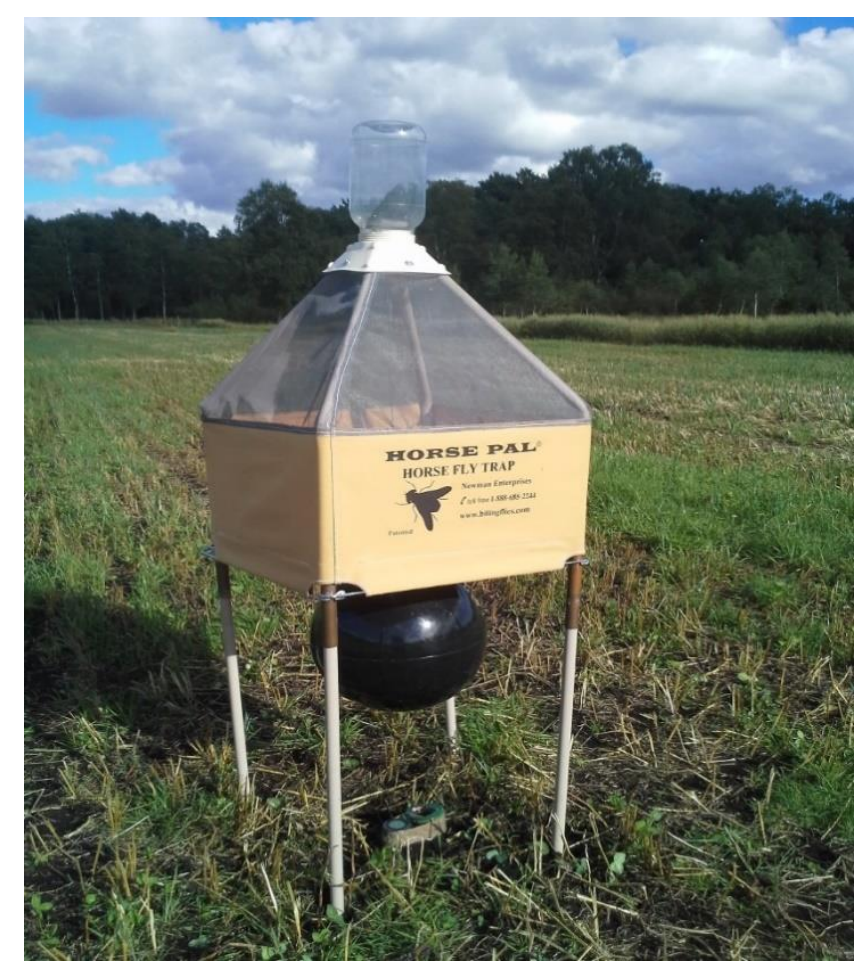

Figure 2. Baited Horse Pal trap set up in Hästhult (wood pasture), Sweden, 2016

\subsection{Study Area}

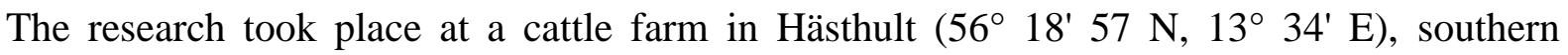
Sweden. The field work, from July 21 to August 31, 2016, was done in a wood pasture with a mixed deciduous-coniferous forest at the back of the traps and on both sides with a few patches of trees. Some of the 125 farm's cows were resting in their shade when they stopped grazing. The grazing area for cows was very large, almost surrounding completely the research area and extending to several nearby places. In the Southeast direction, at about 400 
$\mathrm{m}$, there is a lake and in the Northwest direction, at about $500 \mathrm{~m}$ there is also a large pond.

The Nzi trap was set up for forty - two days in the open area, at a distance of $300 \mathrm{~m}$ from the forest, facing west so that in the afternoon the flies would be attracted to the sunny side of the trap (Mihok et al., 2006; Vaduva, 2016). Horse Pal trap was fixed in the same open area and at a forest distance of $100 \mathrm{~m}$ and $200 \mathrm{~m}$ in a straight line from the Nzi respectively, for the same period of time.

During the experiment, the grass was not allowed to grow inside and around the traps, being mowed when it was necessary.

\subsection{Collection and Specimens Processing}

All tabanids and S. calcitrans (L) were collected daily from 6 to $7 \mathrm{pm}$ during the forty-two days. The individuals of all biting flies were placed in labeled plastic vials and left in a freezer overnight at $-18^{\circ} \mathrm{C}$, to be identified and counted the next day. Both tabanids and stable flies have been identified at the species level as regards the type of used trap and number of specimens collected on the entire period. The species level identification was made in accordance with the description of the species and the keys presented by Chvála et al., (1972); Walker (1994) and Oosterbroek (2006). Each day, before starting the collection of bloodsucking flies, temperature was measured using an environment anemometer LUTRON LM-8000.

\subsection{Field Observations on Both Nzi and Horse Pal Traps With and Without Bait}

Apart from the behavior of the tabanids that landed each time on the blue and black color before entering the Nzi trap, this behavior being specified according to Vaduva (2016), two other observations caught my attention.

These field observations were made two hours a day, between 12 and $14 \mathrm{pm}$ for forty-two days, when it was not cloudy or raining.

a) in connection with the Nzi trap, on all days of observation the first species of tabanids that entered the trap with and without bait, were deer flies, Chrysops species.

b) Horse Pal trap is considered to attract only horse flies, deer flies and yellow fly. In my experiment, I was remarkably surprised to find about thirty-five Syrphidae trapped in the clear jar every day and I do not believe it was accidental. Some species of flies belonging to the Syrphidae family show a preference for the yellow color, such as Episyrphus balteatus, Eristalis tenax and the Horse Pal trap has a similar color in its composition (Lunau, 2014).

I believe that future research could reveal surprising information. The Horse Pal trap should be used in different ecosystems to see what surprises it has to offer.

\subsection{Data Analysis}

In terms of normal distribution, the number of biting flies has been transformed to $\log 10(\mathrm{n}+$ 1) before the computation of variance analyses.

SPSS V22.0 had been used in order to perform the statistical analyses. 


\section{Results}

In this study, the total number of tabanids and S. calcitrans (L) captured in both types of traps was 2178 for tabanid flies and 2631 for stable flies. As regards the tabanid flies, 15 species were caught in unbaited Nzi trap followed by unbaited Horse Pal trap with 14 tabanids species. Likewise, in each of the two baited Nzi and Horse Pal traps were captured 9 species of tabanids. Haematopota pluvialis represented $24.40 \%(\mathrm{n}=154)$ on unbaited Nzi trap and 26.62\% ( $\mathrm{n}=168)$ on baited Nzi trap. With regard to unbaited/baited Horse Pal trap, Haematopota pluvialis was also the most abundant species with $21.30 \%(\mathrm{n}=85)$ and $34.04 \%$ ( $\mathrm{n}=176)$, respectively. Haematopota subcylindrica was the second abundant species captured by unbaited/baited Nzi trap $(14.26 \%, \mathrm{n}=90 / 14.90 \%, \mathrm{n}=94)$ and the third one has been Tabanus bromius species $(12.36 \%, \mathrm{n}=78 / 19.65 \%, \mathrm{n}=128)$ and Tabanus maculicornis caught in great number, especially on baited Nzi trap $13.31 \%(\mathrm{n}=84)$. The second abundant species present in unbaited/baited Horse Pal trap was Tabanus bromius $(13.78 \%, \mathrm{n}=$ $55 / 15.86 \%, \mathrm{n}=82)$ followed by Tabanus maculicornis $16.63 \%(\mathrm{n}=86)$ and Haematopota subcylindrica $15.47 \%(\mathrm{n}=80)$ both species captured in baited Horse Pal trap. In fact, taken separately, Tabanus maculicornis was the second abundant species caught in baited Horse Pal trap and Hybomitra muehlfeldi $10.03 \%(\mathrm{n}=40)$ the third tabanid species caught in unbaited Horse Pal trap (Tables 1-2). In terms of stable flies, S. calcitrans (L) species has been caught in relatively large numbers in the unbaited/baited Nzi trap $(n=832 / 1498)$ and, surprisingly in the unbaited and baited Horse Pal trap $(n=49 / 252)$, but not in such large numbers compared to the Nzi trap (Table 3).

Table 1. Tabanids (number and percentage) collected at Hästhult research location

\begin{tabular}{|c|c|c|c|c|}
\hline \multirow[t]{3}{*}{ Species } & \multicolumn{4}{|c|}{ Nzi trap } \\
\hline & \multicolumn{2}{|c|}{ unbaited } & \multicolumn{2}{|c|}{ baited } \\
\hline & $\mathrm{n}$ & $\%$ & $\mathrm{n}$ & $\%$ \\
\hline Chrysops caecutiens L., 1758 & 15 & $2.38 \%$ & 34 & $5.39 \%$ \\
\hline Chrysops divaricatus Loew, 1858 & 13 & $2.06 \%$ & 32 & $5.07 \%$ \\
\hline Hybomitra bimaculata Macquart, 1826 & 37 & $5.86 \%$ & 0 & $0.00 \%$ \\
\hline Hybomitra ciureai Séguy, 1937 & 45 & $7.13 \%$ & 58 & $9.19 \%$ \\
\hline Hybomitra distinguenda Verrall, 1909 & 14 & $2.22 \%$ & 0 & $0.00 \%$ \\
\hline Hybomitra kaurii Chvála et Lyneborg, 1970 & 27 & $4.28 \%$ & 0 & $0.00 \%$ \\
\hline Hybomitra lundbecki Lyneborg, 1959 & 23 & $3.65 \%$ & 0 & $0.00 \%$ \\
\hline Hybomitra muehlfeldi Brauer, 1880 & 53 & $8.40 \%$ & 0 & $0.00 \%$ \\
\hline Haematopota pluvialis L., 1758 & 154 & $24.40 \%$ & 168 & $26.62 \%$ \\
\hline Haematopota subcylindrica Pandellé, 1883 & 90 & $14.26 \%$ & 94 & $14.90 \%$ \\
\hline Heptatoma pellucens Fabricius, 1776 & 10 & $1.58 \%$ & 16 & $2.54 \%$ \\
\hline Tabanus bovinus L., 1758 & 9 & $1.43 \%$ & 0 & $0.00 \%$ \\
\hline Tabanus bromius L., 1758 & 78 & $12.36 \%$ & 124 & $19.65 \%$ \\
\hline Tabanus glaucopis Meigen, 1820 & 8 & $1.27 \%$ & 21 & $3.33 \%$ \\
\hline Tabanus maculicornis Zetterstedt, 1842 & 55 & $8.72 \%$ & 84 & $13.31 \%$ \\
\hline TOTAL & 631 & $100 \%$ & 631 & $100 \%$ \\
\hline
\end{tabular}


Notes: $\mathrm{n}$, represent the number of individuals in every species; \%, percentage of each species from the total number of collected tabanid specimens. The collection period using unbaited/baited Nzi trap was recorded from July 21 to August 31, 2016.

Table 2. Tabanids (number and percentage) collected at Hästhult research location

\begin{tabular}{|c|c|c|c|c|}
\hline \multirow[t]{3}{*}{ Species } & \multicolumn{4}{|c|}{ Horse Pal trap } \\
\hline & \multicolumn{2}{|c|}{ unbaited } & \multicolumn{2}{|c|}{ baited } \\
\hline & $\mathrm{n}$ & $\%$ & $\mathrm{n}$ & $\%$ \\
\hline Chrysops caecutiens L., 1758 & 0 & $0.00 \%$ & 5 & $0.97 \%$ \\
\hline Chrysops divaricatus Loew, 1858 & 1 & $0.25 \%$ & 2 & $0.39 \%$ \\
\hline Hybomitra bimaculata Macquart, 1826 & 29 & $7.27 \%$ & 0 & $0.00 \%$ \\
\hline Hybomitra ciureai Séguy, 1937 & 37 & $9.27 \%$ & 57 & $11.03 \%$ \\
\hline Hybomitra distinguenda Verrall, 1909 & 7 & $1.75 \%$ & 0 & $0.00 \%$ \\
\hline Hybomitra kaurii Chvála et Lyneborg, 1970 & 24 & $6.02 \%$ & 0 & $0.00 \%$ \\
\hline Hybomitra lundbecki Lyneborg, 1959 & 22 & $5.51 \%$ & 0 & $0.00 \%$ \\
\hline Hybomitra muehlfeldi Brauer, 1880 & 40 & $10.03 \%$ & 0 & $0.00 \%$ \\
\hline Haematopota pluvialis L., 1758 & 85 & $21.30 \%$ & 176 & $34.04 \%$ \\
\hline Haematopota subcylindrica Pandellé, 1883 & 30 & $7.52 \%$ & 80 & $15.47 \%$ \\
\hline Heptatoma pellucens Fabricius, 1776 & 5 & $1.25 \%$ & 11 & $2.13 \%$ \\
\hline Tabanus bovinus L., 1758 & 6 & $1.50 \%$ & 0 & $0.00 \%$ \\
\hline Tabanus bromius L., 1758 & 55 & $13.78 \%$ & 82 & $15.86 \%$ \\
\hline Tabanus glaucopis Meigen, 1820 & 21 & $5.26 \%$ & 18 & $3.48 \%$ \\
\hline Tabanus maculicornis Zetterstedt, 1842 & 37 & $9.27 \%$ & 86 & $16.63 \%$ \\
\hline TOTAL & 399 & $100 \%$ & 517 & $100 \%$ \\
\hline
\end{tabular}

Notes: $n$, represent the number of individuals in every species; \%, percentage of each species from the total number of collected tabanid specimens. The collection period using unbaited/baited Horse Pal trap was recorded from July 21 to August 31, 2016.

Table 3. The number of tabanids and S. calcitrans (L) collected at Hästhult research location

\begin{tabular}{lrrrrr} 
& & \multicolumn{2}{c}{ Nzi trap } & \multicolumn{2}{c}{ Horse Pal trap } \\
\cline { 2 - 6 } & $\begin{array}{c}\text { Captured specimens } \\
\text { (number) }\end{array}$ & unbaited & baited & unbaited & baited \\
\hline Tabanids & 631 & 631 & 399 & 517 \\
\hline S. calcitrans (L) & 832 & 1498 & 49 & 252 \\
\hline
\end{tabular}

Note: the collection period using unbaited/baited Nzi/Horse Pal traps was recorded from July 21 to August 31, 2016.

Considering the total number of tabanids and S. calcitrans (L) captured in Nzi trap, statistical analyzes have shown that the effect of the type of trap (unbaited/baited) on no of tabanids and stable flies seems to be different for both types of species (analysis of variance, ANOVA: $F$ $(1,80)=6.403, P=0.013)$. The presence of interaction tells us that it is reasonable to believe that the difference in average no of tabanids and stable flies captured between the two types of unbaited/baited Nzi trap is not the same for the two types of species. There was also a 
significant main effect of type of species, tabanids/ S. calcitrans (L) on the average no of collected specimens, $P<0.001$ (Figure 3a). As regards the total number of biting flies caught in the second trap, unbaited/baited Horse Pal had a significant influence on the average no of tabanids and $S$. calcitrans (L) as well as the type of species on the average number of collected specimens pointed out by ANOVA: $F(1,80)=7.454, P=0.008 ; F(1,80)=27.360$, $P<0.001$. The interaction was not significant, ANOVA: $F(1,80)=0.523, P=0.472$ (Figure 3b).

(a)

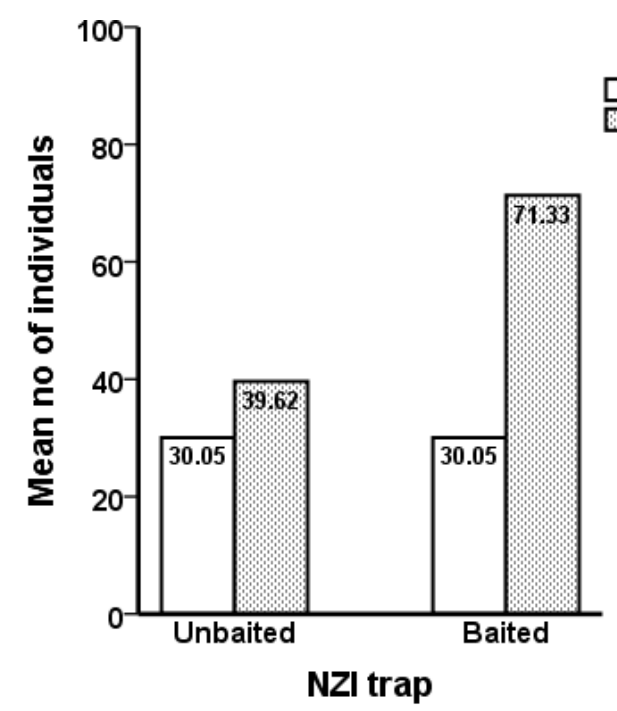

(b)

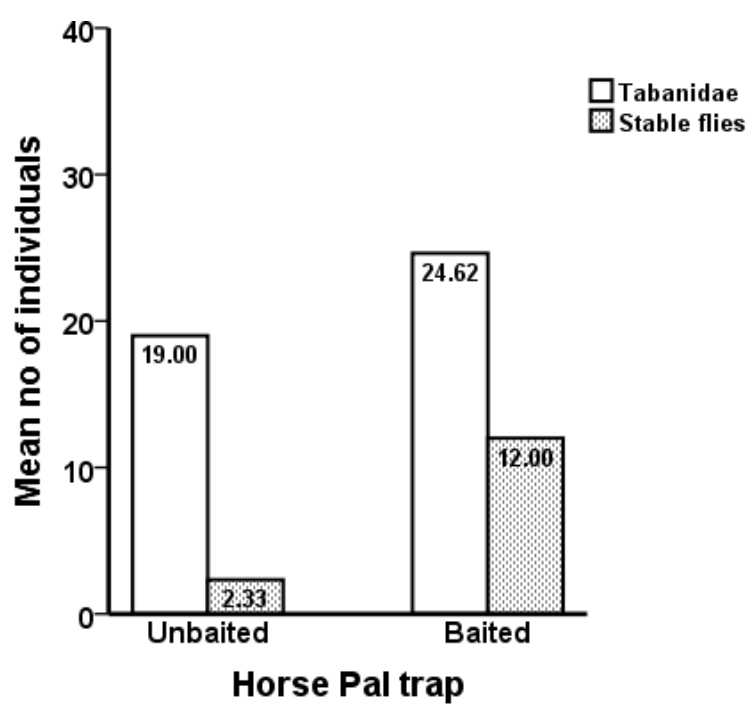

Figure 3. Number of individuals (tabanids/stable flies) captured in both unbaited/baited traps.

The mean number of individuals captured in 42 days in a) unbaited/baited Nzi trap or b) unbaited/baited Horse Pal trap (tabanids: (a) $n=631 / 631$; (b) $n=399 / 517$ ) and (stable flies:

(a) $n=832 / 1498$; (b) $n=49 / 252$ )

Regarding the comparison of the number of biting flies caught in the two types of unbaited traps (Nzi/Horse Pal), the variable type of trap had a significant influence on the average number of tabanids and stable flies by analysis of variance, ANOVA: $F(1,80)=30.431, P<$ 0.001. In contrast, the variable type of species did not have a significant influence on the average number of captured specimens, $P=0.420$. However, the interaction was significant, ANOVA: $F(1,80)=8.968, P=0.004$ suggesting that the difference in no of tabanids and stable flies between both types of species is not the same for both unbaited Nzi and Horse Pal traps (Figure 4a). Moreover, regarding the attractiveness of biting flies at Nzi / Horse Pal with bait, there were statistically significant results such as: the variable type of trap had a significant influence on the average no of tabanids and stable flies, ANOVA: $F(1,80)=$ 37.557, $P<0.001$ and also the variable type of species had significant influence on the average no of collected individuals, ANOVA: $F(1,80)=7.359, P=0.008$. Likewise, the interaction was significant, ANOVA: $F(1,80)=26.020, P<0.001$ pointed out that the effect of type of trap on no of tabanids and S. calcitrans (L) seems to be different for both types of species (Figure $4 b$ ). 
(a)

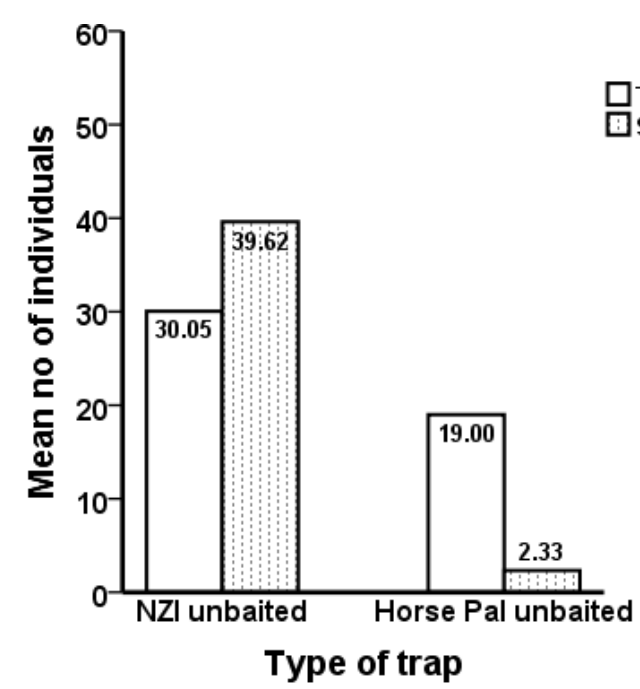

(b)

Figure 4. Comparison of the number of individuals (tabanids and stable flies) caught in both unbaited and baited traps. The numbers displayed in the columns are mean numbers of captured individuals (tabanids/stable flies) during the entire period in a) unbaited Nzi/Horse

Pal traps and also in b) baited Nzi/Horse Pal traps

When it was tested whether the number of tabanids caught in the Nzi with and without bait depended on the type of weather, the result was remarkable. The main effect of unbaited/baited Nzi trap was not significant and so the type of trap had no influence on the average no of tabanids, $P=0.551$. As well as, the interaction was not significant, $P=0.835$. Instead, ANOVA pointed out a significant difference $F(3,34)=26.590, P<0.001$ in terms of the variable type of weather that had influence on the average no of collected tabanids. Tukey HSD, $P<0.001$ revealed a significant difference in the total no of tabanids between two types of weather, Sunny and Cloudy, $P=0.002$ and also the difference was significant for all types of weather and Rainy, $P<0.001$ (Figure 5a). The same type of result was evident for the tabanids caught in the Horse Pal trap with and without bait in relation to the types of weather. The main effect of unbaited/baited Horse Pal trap was also not significant and as well as the interaction, $P=0.270$ and $P=0.796$. There was a significant difference, ANOVA: $F(3,34)=19.489, P<0.001$ with regard to the variable type of weather and the influence on the average number of captured tabanids. Tukey HSD, $P<0.001$ showed the same significant difference between Sunny and Cloudy, $P=0.015$ and for all types of weather and Rainy, $P<$ 0.001 (Figure 5b). 


\section{Ml Macrothink}

(a)

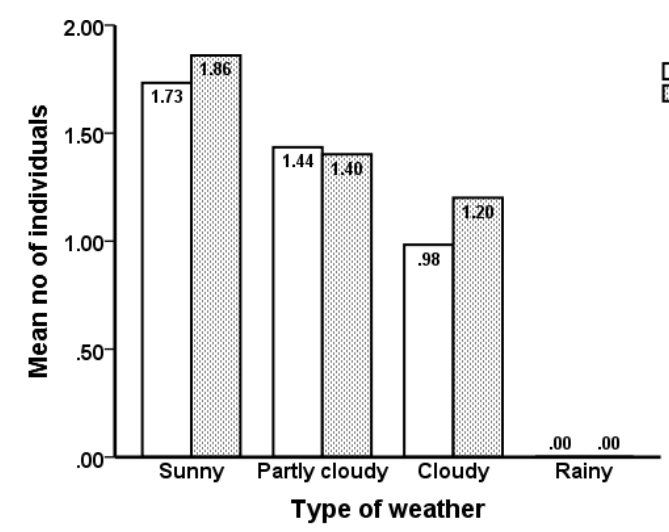

Journal of Biology and Life Science

ISSN 2157-6076

2020, Vol. 11, No. 2

(b)

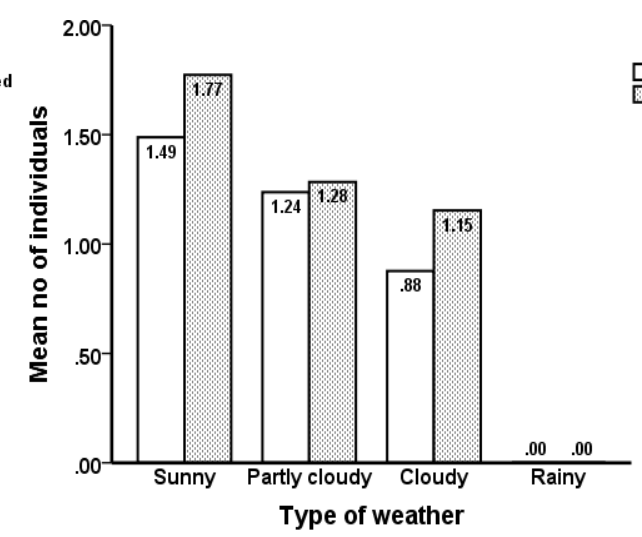

Figure 5. The tabanid species captured according to all types of weather \& types of traps

The mean number of tabanids that were caught in unbaited/baited Nzi trap (a) and unbaited/baited Horse Pal trap (b). All the numbers displayed in the columns represent the mean numbers of captured tabanid individuals on different types of weather in each of the two traps with or without bait.

When analyzing the hypothesis whether the number of $S$. calcitrans (L) caught in the unbaited/baited Nzi trap depended on the type of weather, ANOVA showed significant results such as the variable type of trap (unbaited/baited) had influence on the average no of stable flies $F(1,34)=28.970, P<0.001$ and also the presence of interaction tells us that it reasonable to believe that the difference in no of $S$. calcitrans (L) between all types of weather is not the same for the Nzi trap with or without bait, $F(3,34)=3.439, P=0.028$. Likewise, the variable type of weather had influence on the average no of collected individuals, $F(3,34)=24.828, P<0.001$. Tukey HSD, $P<0.001$ pointed out a significant difference between Sunny and Cloudy, $P=0.020$ and for all types of weather and Rainy, $P<$ 0.001 (Figure 6a). Almost the same pattern in the result was observed from statistical analyzes regarding the attraction of $S$. calcitrans (L) to unbaited/baited Horse Pal depending on the weather. The main effect for trap and also the main effect for type of weather were qualified, however, by a significant interaction between the two factors, $F(1,34)=36.307, P$ $<0.001$ and $F(3,34)=11.618, P<0.001$. Tukey HSD, $P<0.001$ revealed a significant difference between all types of weather and Rainy, $P<0.001$. The interaction was not significant, $P=0.076$ (Figure 6b). 
(a)

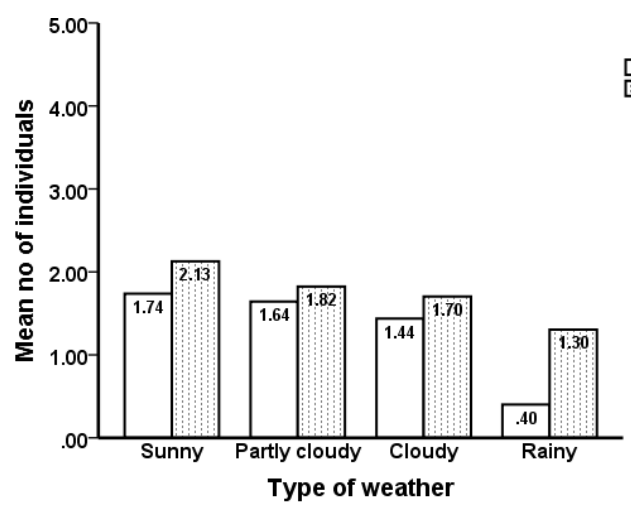

(b)

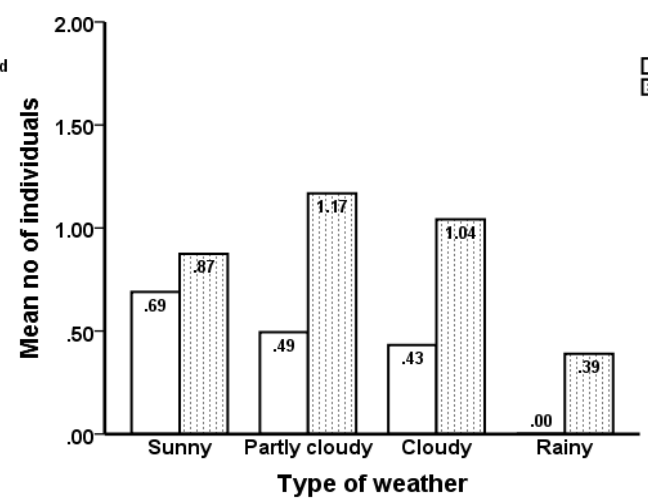

Figure 6. S. calcitrans (L) species captured according to all types of weather \& types of traps The mean number of $S$. calcitrans (L) that was caught in unbaited/baited Nzi trap (a) and unbaited/baited Horse Pal trap (b). All the numbers displayed in the columns represent the mean numbers of captured S. calcitrans (L) individuals on different types of weather in each of the two traps with or without bait

\section{Discussion and Conclusions}

This study continued my published research work on the efficiency of the Nzi trap in Sweden in capturing tabanids and stable flies, but this time the trap was baited with two different attractants such as aged urine and acetone. I chose the same type of trap such as Nzi, as in the previous experiment by Vaduva (2016) and a new trap, Horse Pal, which was tested by this study for the first time in Scandinavia and maybe even in Europe and also the same suitable area for conducting the experiments, such as wood pasture with mixed coniferous/deciduous forest. Wood pasture gave the higher catches of biting flies, especially tabanids with frequently large captures of Tabanus bromius (Brightwell and Dransfield, 2014). In addition, in the second part of the experiment, the efficiency of the trap / odor-bait relationship was tested. Likewise, in order to determine whether odor traps could provide a useful means of capturing as many species of tabanids as possible and as many individuals belonging to those species in that area, as well as specimens of stable flies, a comparison was made between the traps with olfactory attractants and those without them. For biting flies as well as other insects, among other mechanisms, visual cues and odors are the most important factors in long range orientation and host location (Gibson and Torr, 1999). After many behavioral observations on tabanids and stable flies in the field, I could confirm that sometimes they approach the trap and when they get very close do not seem to be attracted to it and sheer off. However, the blue and black panels on the Nzi trap proved to be very effective in attracting tabanids due to the strong intensity of the contrast of the trap in relation to its immediate environment. Field observations showed that tabanid species landed on black surfaces, preferring the famous blue color (Gibson and Torr, 1999; Lehane, 2005; Vaduva, 2016). The contrast against the background provided by blue and black panels of the Nzi trap, act as an important signal in the detection and orientation of biting flies to trap (Allan et al., 1987). Likewise, the 125 existing cattle on the farm emit the right signal to activate the biting flies in the immediate vicinity or even at a certain distance. The nearby natural host is an essential factor in the attractiveness and 
performance of the traps. The tabanids species such as Tabanus bromius and Tabanus maculicornis are extremely sensitive to aged cow urine used as attractant (Krčmar et al., 2006). Phenolic attractants occur naturally in bovine urine and also following on from other experiments there was suggested that unidentified attractants are present in the odor of cattle (Torr et al., 2006).

The Nzi trap failed to increase the captures of tabanids from trap with lures and surprisingly, unexpected and probably unusually, the caught number of tabanids was identical in both types unbaited and baited Nzi trap. This result highlighted again the idea that the attractiveness of the Nzi trap to tabanids is not significantly reduced when an attractant is not used (Vaduva, 2016).

When comparing the Horse Pal trap with and without bait, it was found that the number of tabanids caught was different, but not significantly different, suggesting that this type of trap is as effective in catching tabanids as the Nzi trap, and it would probably have been more or less effective if it had been tested in the vicinity of horses just like in North America (Watson et al., 2007). There were caught several tabanids in the Horse Pal trap with bait, as the tabanids were attracted by their dependence on the amount of odor released even from greater distances (Lehane, 2005). The Nzi trap has been tested near the horses and also cows in Sweden (Vaduva, 2016).

As regards the number of tabanids caught by both the unbaited and baited Horse Pal trap, the number was lower than in the Nzi trap as well as with or without bait, but it did not differ significantly between the two types of traps. The Nzi trap attractiveness to biting flies, especially tabanids has been pointed out by a plenty of field experiments because the black and white (with the strong blue reflectivity) colors presence in its manufacture make a great contrast on background and the blue color gives the maximum contrast (Gibson and Torr, 1999; Lehane, 2005).

When Nzi trap was baited with cattle urine and acetone, the trap significantly increased stable flies catches relative to the Nzi trap without attractant lures added. The number of tabanids was significantly lower in baited Nzi trap, compared to $S$. calcitrans (L) probably due to the fact that some species of tabanids may react differently in terms of the attractiveness to the type of attractant. As a surprise was the fact that Nzi baited trap was very attractive to $S$. calcitrans (L) There was a significant difference due to the stable flies are strongly attracted to the odors, because their antennae contain the olfactory senses that respond to the host environment in relation to the associated odors. The others explanations would be that I used aged cow urine as bait in combination with acetone and traps with binary blend lures caught more stable flies in the field experiments. The field observations revealed that the number of $S$. calcitrans (L) increased significantly in meadows or field crops when cattle manure was applied (Tangtrakulwanich et al., 2015). In this area, the owner of the farm applied this treatment every year, which did not happen in the two locations where my previously published research work took place. Following the result, these explanations lead to the understanding of the large number of S. calcitrans (L) caught in the bait Nzi trap and the relatively large number caught in the bait Horse Pal trap. The two types of attractants, acetone 
and aged urine in association with manure applied annually can act as chemical cues suggesting the presence of cows in the area (Tangtrakulwanich et al., 2015). Furthermore, the significantly small number of S. calcitrans (L) caught in Horse Pal trap with bait compared to baited Nzi trap suggests that this type of trap is not ideal in attracting stable flies as has already been specified by the manufacturers of this type of trap. In addition, S. calcitrans (L) was caught in the Horse Pal trap without the bait likely by chance, as these flies use the shaded resting sites as places where they go in order to digest their blood meal. So, probably, the Horse Pal trap served as in ideal resting site and ultimately, become more or less attractive to flies (Lehane, 2005). To meet the energy requirements and the development of eggs, stable flies need several blood meals. Seemingly, a unique characteristic of $S$. calcitrans (L) is the utilization of blood in order to get triacylglycerol synthesis and also utilize sucrose in glycogen synthesis (Venkatesh and Morrison, 1982).

Weather, especially temperature, can play a regulatory role in the tabanids and stable flies peak emergence. The average temperature on sunny days was $23.2^{\circ} \mathrm{C}$ and on rainy days $14.9^{\circ} \mathrm{C}$, which made the tabanids unable to fly. In terms of stable flies, the individuals were caught in the first days with rain. The number of tabanids caught in the Nzi or Horse Pal traps with or without bait depended on the types of weather and not on the type of trap. Likewise, the number of tabanids differed between the two types of weather such as sunny and cloudy and between all types of weather and rainy (Figures 5a, 5b). With respect to cloudy weather, the capture of tabanids has suffered a significant reduction and also the rainy days have led to zero capture of flies. Instead, for S. calcitrans (L) the type of trap (Nzi unbaited / baited) as well as the type of weather had an influence on the number of stable flies collected. The difference in the type of weather and the number of individuals of $S$. calcitrans (L) collected was between sunny and cloudy and again between all types of weather and rainy.

In terms of the attraction of S. calcitrans (L) to Horse Pal trap with or without bait depending on the type of weather, the result was similar but the difference in the number of stable flies captured was observed only between all types of weather and rainy (Figures 6a, 6b).

In the two consecutive periods of the project, without and with bait, the number of sunny and partly cloudy days was identical and the type of species was almost identical, as the research covered the entire period of full activity of the most of these species. The missing species in both types of bait traps were Tabanus bovinus, Hybomitra muehlfeldi, Hybomitra lundbecki, Hybomitra kaurii, Hybomitra distinguenda, Hybomitra bimaculata. Although species that were absent from bait traps should theoretically no longer be active before August, but they were still present in relatively large numbers in the unbaited traps before August 11. Probably, they ceased to exist after that period, because they have become absent in the bait traps or could be a selective pressure for niche differentiation between tabanids species with respect to the period of activity (Brightwell and Dransfield, 2014). One species of tabanids that was missing from the unbaited Horse Pal trap was Chrysops caecutiens, although this species is active until September. It lives especially near water and the trap needs to be generally setted nearly cattle (Chvála et al., 1972). The second condition was met, and the first one more or less, taking into account the fact that the pond and the lake are not located too far and yet it was not attracted to the Horse Pal trap. As regards the Chrysops caecutiens and Chrysops 
divaricatus species the number of individuals caught by Nzi trap was relatively small compared to the other species of caught tabanids and did not differ significantly in the Nzi bait trap, probably due to the fact that these species known as deer flies are resting most of the time and do not have an active search behavior. In particular, these species of tabanids pick up scents from the air when animals are nearby and alternately, the odor of bait traps acts as a signal in activating the flight in order to obtain the daily blood meal (Allan et al., 1987).

Due to their habit of obtaining a daily blood meal and associated with the transmission of various diseases, tabanids and stable flies can sometimes become serious problems for animals and just as well for humans. Tabanids are very good fliers and extremely active on warm and sunny days. For example, Haematopota pluvialis, which was caught in large numbers even on cloudy days, is a species of tabanids that flies silently and of whose presence you are only aware when it bites you. Furthermore, Tabanus bromius being a fairly large species of horse flies and being caught in relatively large number together with Haematopota subcylindrica, Tabanus maculicornis, Hybomitra muehlfeldi and Hybomitra ciureai could become the real pests by their painful bite in the research location, especially for existing ruminants who are the favorite hosts through their slow movement over longer or shorter distances when they are feeding. The result of this work might provide an objective basis for investigating further the chemical sense of tabanids and S. calcitrans (L) since they may be tested in association with different attractants. However, the efficiency of the Nzi and Horse Pal traps in catching of biting flies should be also tested in other ecosystems on the best time that corresponds to maximum activity of flies and also in relation with other animals and different attractants in order to understand the hunting patterns of tabanids and stable flies.

\section{Acknowledgments}

I thank Anders Svensson for the permission to run the research work on his property and for supplying the cow urine and also Denis Vaduva for his invaluable help in the field. I am profoundly grateful to Professor Eric Warrant for his agreement and support in purchasing the two Horse Pal traps and also to the three anonymous readers of the manuscript for their valuable feedback. This study was supported by a grant from "Entomologiska Sällskapet i Lund" (to G. V.) and I would like to thank Mikael Sörensson in this respect.

\section{References}

Allan, S. A., Day, J. F., \& Edman, J. D. (1987). Visual Ecology of biting flies. Annual Review of Entomology, 32, 297-316. https://doi.org/10.1146/annurev.en.32.010187.001501

Axtell, R. C. (1976). Coastal horse flies and deer flies (Diptera: Tabanidae). North - Holland Publishing Company, p. 416.

Brightwell, R., \& Dransfield, R. (2014). Survey of Tabanidae (horseflies) in southern England 2014. InfluentialPoints, p. 1.

Chvála, M., Lyneborg, L., \& Moucha, J. (1972). The horse flies of Europe (Diptera, Tabanidae). Copenhagen: Entomological Society of Copenhagen

Foil, L. D., \& Hogsette, J. A. (1994). Biology and control of tabanids, stable flies and horn 
flies. Revue Scientifique et Technique (International Office of Epizootics), 13, 1125-1158. https://doi.org/10.20506/rst.13.4.821

Gibson, G., \& Torr, S. J. (1999). Visual and olfactory responses of haematophagous Diptera to host stimuli. Medical and Veterinary Entomology, 13, 2-23. https://doi.org/10.1046/j1365-2915.1999.00163.x

Hogsette, J. A., Ruff, J. P., \& Jones, C. J. (1987). Stable Fly Biology and Control in Northwest Florida. Journal of Agricultural Entomology, 4(1), 1-11.

Kingdon, J. (1982). East African Mammals. An Atlas of Evolution in Africa. vol IIIC, Bovids. London: University of Chicago Press edition 1989. London: Academic Press (Inc.) London Ltd.

Krčmar, S., Mikuska, A., \& Merdić, E. (2006). Response of Tabanidae (Diptera) to different natural attractants. Journal of Vector Ecology, 31, 262-265. https://doi.org/10.3376/1081-1710(2006)31[262:ROTDTD]2.0.CO:2

Lehane, M. J. (2005). The Biology of Blood-Sucking in Insects ( $2^{\text {nd }}$ edition). Cambridge: Cambridge University Press. https://doi.org/10.1017/CBO9780511610493

Liénard, E., Salem, A., Jacquiet, P., Grisez, C., Prévot, F., Blanchard, B., .. Franc, M. (2013). Development of a protocol testing the ability of Stomoxys calcitrans (Linnaeus, 1758) (Diptera: Muscidae) to transmit Besnoitia besnoiti (Henry, 1913) (Apicomplexa: Sarcocystidae). Parasitology Research, 112, 479-486. https://doi.org/10.1007/s00436-012-3157-6

Lunau, K. (2014). Visual ecology of flies with particular reference to colour vision and colour preferences. Journal of Comparative Physiology A, 200, 497-512. https://doi.org/10.1007/s00359-014-0895-1

Mihok, S. (2002). The development of a multipurpose trap (the Nzi) for tsetse and other biting flies. Bulletin of Entomological Research, 92, 385-403. https://doi.org/10.1079/BER2002186

Mihok, S., Carlson, D. A., Krafsur, E. S., \& Foil, L. D. (2006). Performance of the Nzi and other traps for biting flies in North America. Bulletin of Entomological Research, 96, 387-397.

Mullens, B. A. (2002). Horse Flies and Deer Flies (Tabanidae). Medical and Veterinary Entomology. https://doi.org/10.1016/B978-012510451-7/50015-3

Ndegwa, P. N., \& Ogodo, J. A. (2002). Community structure and diel activity patterns of Stomoxyinae from odour-baited Nzi trap collections. Insect Science \& it's Application, 22, 275-280. https://doi.org/10.1017/S1742758400020890

Oosterbroek, P. (2006). The European Families of the Diptera Identification, diagnosis, biology. Utrecht: KNNV Publishing. https://doi.org/10.1163/9789004278066

Tangtrakulwanich, K., Albuquerque, T. A., Brewer, G. J., Baxendale, F. P., \& Zurek, L. 
(2015). Behavioural responses of stable flies to cattle manure slurry associated odourants. Faculty Publications: Department of Entomology, 375. https://doi.org/10.1111/mve.12103

Torr, S. J., Mangwiro, T. N. C., \& Hall, D. R. (2006). The effects on host physiology on the attraction of tsetse (Diptera: Glossinidae) and Stomoxys (Diptera: Muscidae) to cattle. Bulletin of Entomological Research, 96, 71-84. https://doi.org/10.1079/BER2005404

Vaduva, G. (2016). A study on bloodsucking Tabanidae and Stomoxys calcitrans (Diptera) attacking horses and cows in Northern Scania, Sweden. Journal of Biology and Life Science, 7. https://doi.org/10.5296/jbls.v7i1.8611

Van Hennekeler, K., Jones, R. E., Skerratt, L. F., Fitzpatrick, L. A., Reid, S. A., \& Bellis, G. A. (2008). A comparison of trapping methods for Tabanidae (Diptera) in North Queensland, Australia. Medical and Veterinary Entomology, 22, 26-31. https://doi.org/10.1111/j.1365-2915.2007.00707.x

Venkatesh, K., \& Morrison, P. E. (1982). Blood Meal as a Regulator of Triacylglycerol Synthesis in the Haematophagous Stable Fly, Stomoxys calcitrans. Journal of Comparative Physiology, 147, 49-52. https://doi.org/10.1007/BF00689289

Walker, A. (1994). Arthropods of humans and domestic animals. A guide to preliminary identification. London: Chapman \& Hall

Watson, D. W., Denning, S. S., Calibeo - Hayes, D. I., Stringham, S. M., \& Mowrey, R. A. (2007). Comparison of Two Fly Traps for the Capture of Horse Flies (Diptera: Tabanidae).

Journal of Entomological Science, $\quad 42(2), \quad$ 123-132. https://doi.org/10.18474/0749-8004-42.2.123

Wood, D. M. (1985). Biting flies attacking man and livestock in Canada. Agriculture Canada, Publication 1781/E. https://doi.org/10.5962/bhl.title.59471

\section{Copyright Disclaimer}

Copyright for this article is retained by the author(s), with first publication rights granted to the journal.

This is an open-access article distributed under the terms and conditions of the Creative Commons Attribution license (http://creativecommons.org/licenses/by/4.0/). 